\title{
Overview on Covid-19 Vaccine - A Short Review
}

\author{
Aditi Sharma ${ }^{1}$, K. K. Sharma ${ }^{2}$ \\ ${ }^{1}$ Department of Community Medicine, SPH PGI, Chandigarh, India. \\ ${ }^{2}$ Department of Biochemistry, Dr YSPGMC, Nahan, Himachal Pradesh, India.
}

\section{ABSTRACT}

\section{BACKGROUND}

COVID-19 is a serious disease caused by a novel coronavirus known as SARS-CoV-2 (severe acute respiratory syndrome). With a population of 1.38 billion people, India aimed to vaccinate 300 million people in the first phase of the COVID-19 vaccination program by August 2021, including 30 million health workers and frontline workers, as well as 270 million elderly people (over 50 years) and those people with co-morbidities. COVID-19 vaccination has begun in India with two types of vaccines: Covishield (by Serum Institute of India Ltd) and Covaxin (by Bharat Biotech International Ltd), according to the Press Information Bureau. Manufacturers in India have said that they will be able to meet the country's future COVID-19 vaccination needs. Both the vaccines function by priming the immune system with a SARS-CoV-2 spike protein and require two doses. Covaxin uses an inactivated SARS-CoV-2 virus taken from an asymptomatic patient, while Covishield uses a weakened variant of adenovirus. On prime minister Narendra Modi's birthday, which is on the 17th of September 2021, a massive immunization blitz is planned. With the CoWIN website updated every second throughout the day, India has administered 2.25 crore Covid-19 vaccine doses.

\section{METHODS}

The review study was carried out in PGI Chandigarh, with the base of the material accessed online and a few studies conducted recently. We conducted a literature search using the MEDLINE electronic database to identify published studies until Sep 2021. The search was confined to peer-reviewed articles that were published in English and contained an abstract. Reference lists of journal articles were also screened for additional citations fitting our search criteria.

\section{CONCLUSIONS}

We conclude that masks alone are insufficient to provide adequate protection against COVID-19 and that they must be used in conjunction with physical distancing, hand cleanliness, and vaccination to put an end to the global SARS-CoV-2 pandemic. A successful vaccination is safe, efficacious, long-lasting, and deployable to large populations, and one should follow the local health authority's recommendations.

\section{KEY WORDS}

Coronavirus, Vaccine development, Vaccination, Prevention.
Corresponding Author: Dr. Aditi Sharma

Senior Research Fellow, Department of Community Medicine, SPH, PGI, Chandigarh-160014, India. E-mail: aaditisharmabk@gmail.com

DOI: $10.14260 / j e m d s / 2021 / 814$

How to Cite This Article: Sharma A, Sharma KK. Overview on Covid19 vaccine - a short review. J Evolution Med Dent Sci 2021;10(45):4032-4036, DOI: $10.14260 /$ jemds $/ 2021 / 814$

Submission 26-10-2021,

Peer Review 02-12-2021, Acceptance 09-12-2021, Published 31-12-2021.

Copyright (C) 2021 Aditi Sharma et al. This is an open access article distributed under Creative Commons Attribution License [Attribution 4.0 International (CC BY 4.0)] 


\section{BACKGROUND}

Covid-19 was first discovered in Wuhan, China, at the end of 2019 and is now affecting people all over the world. On January 13, 2020, Thailand registered the first imported case of COVID-19. Two days later, a second case was discovered in Japan. The third country to report an imported COVID-19 instance was South Korea. On 12 January 2020, the World Health Organization (WHO) briefly named the virus as the novel coronavirus (2019- nCoV), and on 12 February 2020, formally named this infectious coronavirus disease 2019 (Covid-19).

Based on phylogeny, taxonomy, and previous practice, the causal agent was later officially named SARS-CoV-2 by the International Committee for Virus Taxonomy (ICTV). On March 11, 2020, the World Health Organization labelled the outbreak as a pandemic.1-3

\section{DEVELOPMENT OF VACCINES}

\section{mRNA Vaccines}

The mRNA-based vaccination is a new, non-infectious, nonintegrating platform that has a lower risk of insertional mutagenesis. Because of its low cost and high safety profile in animal trials, it is the most promising option.

The immunogenicity of the mRNA can be lowered, and the vaccine's stability can be improved. Anti-vector immunity can also be minimized because mRNA is the least immunogenic genetic vector, allowing the vaccine to be employed multiple times. This platform has benefitted the quick vaccine development effort due to its flexibility and ability to mimic antigen structure and expression as seen in real infection. 4,5

Pfizer and Moderna's novel messenger RNA (mRNA) vaccines are two of the vaccines with the earliest Phase III results. Both presented preliminary data that claimed efficacies of above $90 \%$. They work by injecting mRNA encoding the SARS-CoV-2 spike protein directly into the host. Although pure mRNA is rapidly degraded, technical advances in delivery systems and RNA carriers have allowed for the speedy and safe transport of mRNA into the cytosol, where ribosomes convert the mRNA into a viable protein that can then stimulate an immune response. This innovation has several theoretical advantages over more traditional vaccine types, including enhanced safety (because no infectious agents are used in their production), low mutation risk, reduced risk of antigen degradation in vivo, and the potential for rapid mass production at a lower cost, because in vitro reactions can quickly generate high yields of the therapeutic agent. ${ }^{6}$

\section{Virus Vector Vaccines}

The vaccine against viral vectors is a promising prophylactic solution. These vaccines transfer genes to target cells in a precise manner, are highly efficient in gene transduction, and elicit an immunological response. Viral vector vaccines have a longer half-life and higher antigenic protein expression, making them more effective for prophylaxis because they activate cytotoxic $\mathrm{T}$ cells, which kill virus-infected cells.
Replicating (measles virus and vesicular stomatitis virus) and non-replicating vectors like adenoviruses (Ad) and poxviruses are the two types of viral vectors. A nonreplicating Ads vector is used in the creation of several vaccines. These vectors are physically and genetically stable and do not integrate into the host DNA, making them reasonably safe. Ad vector can infect DCs and dividing or nondividing cells. Ad vectors, on the other hand, require high doses to elicit an immunological response from the host.7,8 AZD1222 (ChAdOx1 nCoV-19) is a SARS-CoV-2 vaccine candidate that uses a non-replicating chimp adenovirus (ChAdOx1) as a vector and has been engineered to generate the S protein from SARS-CoV-2. The University of Oxford and AstraZeneca are working together to develop AZD1222. This adenovirus has been genetically altered to prevent it from replicating in humans. ${ }^{9}$ Cells produce spike protein after vaccination, and the crucial immune system produces neutralizing antibodies that attach to spike glycoprotein and target the SARS-CoV-2 virus. Furthermore, the vaccination activates T-cells, which can target host cells infected with the SARS-CoV-2 virus. After the announcement of Sputnik-V, the Russian government got preliminary orders for more than 1 billion doses of the Sputnik V vaccine from 20 countries. Ad5$\mathrm{nCoV}$ and CoroFlu are in preclinical trials, and Sputnik V has shown antibody generation in all study participants. Russia is now producing around 500 million doses of the Sputnik V vaccine. ${ }^{10}$

\section{Adjuvanted Protein Vaccines}

NVX-CoV2373 is a nanoparticle vaccine made up of trimeric full-length SARS-CoV-2 spike glycoproteins and Matrix-M1 adjuvant. rSARS-CoV-2 is a recombinant nanoparticle vaccine developed by Novavax and manufactured by Emergent Biosolutions. It is made from the full-length (i.e. including the transmembrane domain) wild-type SARS-CoV-2 spike glycoprotein (GenBank accession number, MN908947; nucleotides 21563-25384) optimized in the established baculovirus Spodoptera frugiperda (Sf9) insect cellexpression system.

The 682-QQAQ-685 mutations at the S1/S2 cleavage sites confer protease resistance; while two proline changes at residues K986P and V987P at the top of the heptad repeat $1 /$ central helix in the S2 subunit maintain the construct in a prefusion conformation. rSARS-CoV-2 is resistant to proteolytic cleavage, binds to the hACE2 receptor with high affinity, and is thermostable. Novavax manufactured MatrixM1, which is a saponin-based adjuvant. Both the vaccination and the adjuvant were kept at temperatures ranging from 2 to 8 degrees Celsius. The placebo was 0.9 percent normal saline, which was sterile. ${ }^{11-13}$

\section{DNA Vaccines}

Vaccines made from DNA are the most recent advancement in vaccine development. Using recombinant DNA technology, vaccines are created through genetic alteration. Through a plasmid or viral vector, the DNA encoding the target molecule is transferred into a suitable microorganism or cell line, where it is produced and translated into protein. To recover the product, extraction and purification are utilized. A 
plasmid with a promoter that increases the synthesis of immunogenic proteins is injected. ${ }^{14}$

\section{Inactivated Vaccines}

Killed vaccines, also known as inactivated vaccines, are created by growing the virus in a culture medium and then killing it with chemicals, heat, or radiation. Vaccines against hepatitis A, polio, and measles are examples of this type of vaccine. The viruses in these vaccines can't reproduce in immune-compromised people, so there's no risk of infection. They are ineffective compared to live-attenuated vaccines, eliciting mostly an antibody-mediated immune response with low cell-mediated immunity; need many doses to achieve full immunity. Inactivated vaccine platforms have been widely employed throughout the previous 70 years. Viruses are inactivated using chemicals, UV light, and heat, resulting in inactivated vaccines. The inactivation of the bacterium results in a safe vaccine, especially for immunocompromised persons. These vaccines, on the other hand, provide a weaker immune response than live vaccines and necessitate more booster doses. Inactivated vaccines take longer to create because the virus must be cultured in the lab before being inactivated.15,16 CoronaVac, formerly known as PiCoVacc, is a candidate-inactivated vaccine produced by Sinovac Biotech, one of China's leading vaccine manufacturers. The CoronaVac vaccine is made by growing viruses in cell culture and then killing them with formalin and an alum adjuvant. The RBD in inactivated SARS-CoV-2 viruses' $\mathrm{S}$ protein works as an immune inducer. ${ }^{16}$ Wuhan Institute of Biological Products and Sinopharm have developed New Crown COVID-19, an inactivated whole-virus, alum-adjuvant vaccine. Before being adsorbed on $0.5 \mathrm{mg}$ alum, the whole virus was inactivated in vitro. BBIBP-CorV, another inactivated vaccine candidate, was developed by the Beijing Institute of Biological Products and Sinopharm. In preclinical experiments, BBIBP-CorV produced a superior immune response to protect against SARS-CoV-2 in guinea pigs, mice, rats, rabbits, and nonhuman primates. BBIBP-CorV was proven to be safe and well-tolerated in phase I and II trials on days 0 and 28 at all three dosages $(2 \mathrm{~g}$, $4 \mathrm{~g}$, or $8 \mathrm{~g}$ ). A significant immune response was observed in $100 \%$ of the vaccine participants. A phase III trial of BBIBPCorV is underway in Abu Dhabi, UAE. ${ }^{17}$ Covaxin is India's first inactivated vaccine candidate against COVID-19, developed by Bharat Biotech, the Indian Council of Medical Research, and the National Institute of Virology. The vaccine-induced robust immune responses, halting the transmission of the SARS-CoV-2 virus. ${ }^{18}$

\section{Live Attenuated Vaccines}

Several infectious disease epidemics including yellow fever, mumps, measles, rubella, polio, and chickenpox, were eliminated by live-attenuated vaccinations (LAV). Recombinant variants of the S protein are encoded by the highly attenuated modified vaccinia virus Ankara, recombinant adenoassociated virus, or attenuated parainfluenza virus. ${ }^{19}$ SARS-CoV-2 LAV vaccines are now in the preclinical stage of research. For example, Indian Immunologicals Limited is collaborating with Griffith University to create a vaccine against SARS-CoV-2 that uses codon deoptimization as a method. Following a single injection, the vaccine candidate confers long-lasting immunity against SARS-CoV-2.20 DelNS1-SARS-CoV2-RBD is a vaccination strain based on influenza that lacks the pathogenic NS1 gene. The immune antagonist and a pathogenic element are both deleted, which reduce the severity of the infection. It is more immunogenic than the wild-type influenza virus and can be administered as a nasal spray. The RBD domain of the SARS-CoV-2 spike protein was encoded on the surface of an attenuated influenza virus. ${ }^{21}$

\section{VACCINATION IN INDIA}

In India, free immunization against COVID-19 began on January 16, 2021, and the government is asking all of its citizens to get vaccinated as part of what is believed to be the world's largest vaccination program. Four of the eight COVID19 vaccines currently undergoing clinical trials in India were created here. With a population of 1.38 billion people, India aimed to vaccinate 300 million people in the first phase of the COVID-19 vaccination program by August 2021, including 30 million health workers and frontline workers (e.g., police, soldiers), as well as 270 million elderly people and people with co-morbidities. According to the Press Information Bureau, COVID-19 vaccination in India has been initiated with two types of vaccines: Covishield (by Serum Institute of India Ltd) and Covaxin (by Bharat Biotech International Ltd). Indian manufacturers have stated that they can meet the country's future needs for COVID-19 vaccines. ${ }^{22,23}$

\section{The Major Vaccines and Vaccine Candidates Developed and/or Manufactured in India. ${ }^{24}$}

Bharat Biotech collaborated with the ICMR and the NIV to produce Covaxin (BBV 152), which has an effectiveness of 80.6 percent (interim analysis) (Whole-virion inactivated Vero cell-derived platform). An emergency use authorization (EUA) with a manufacturing capacity of 150 million doses per year was obtained in India. Sri Lanka, Mongolia, Myanmar, Bahrain, Oman, the Philippines, Maldives, and Mauritius have all received supply through government-to-government agreements. Ocugen and Bharat Biotech have agreed to collaborate on the development and production of Covaxin for the US market.

Covishield (AZD1222) with an efficacy of $70.4 \%$ was developed by Oxford University, AstraZeneca and Serum Institute of India (SII). Vaccine Platform is by a replicationdeficient adenoviral vectored vaccine. In India, a EUA was granted. Its manufacturing capacity is $70-100$ million doses per month. SII is selling the vaccine for less than $\$ 3$ per dose, making it the world's cheapest COVID-19 vaccine.

Gamaleya National Research Institute of Epidemiology and Microbiology produced Sputnik V (Gam-COVID-Vac), a 91.6 percent effective recombinant adenovirus vaccine (rAd26 and rAd5). Dr Reddy's Laboratories, Virchow Biotech, Stelis Biopharma, Gland Pharma, Hetero Biopharma, and Panacea Biotech are the manufacturers in India. RDIF signed production contracts with Virchow Biotech (200 million), Stelis Biopharma (200 million), Gland Pharma (252 million), Hetero Biopharma (100 million), and Panacea Biotec (100 million) doses per year. Sputnik $V$ vaccine will be supplied in India by Dr Reddy's Laboratories and RDIF under a 
production and distribution arrangement (250 million doses per year).

Biological E Limited in India manufactures Janssen Ad26.COV2.S (JNJ-78436735), a 66.1 percent effective replication-incompetent adenovirus (Ad26) vectored vaccine developed by Johnson \& Johnson (Janssen Pharmaceuticals). To expand their manufacturing capacities, Johnson \& Johnson and Biological E Limited have agreed to share technology. By the end of 2022, a deal has been negotiated to produce 1 billion vaccine doses. The vaccine platform for Covovax (NVXCoV2373) is a recombinant spike protein nanoparticle vaccine, which has an effectiveness of 89.3 percent. Novavax developed it with CEPI support, and the Serum Institute of India manufactures it in India. SII has been permitted to manufacture and distribute the vaccine in low- and middleincome countries, including India. Novavax and SII have also committed to supplying the COVID-19 Vaccine with Global Access (COVAX) facility for with 1.1 billion doses.

Zydus Cadila, India developed and manufactured the ZyCoV-D DNA vaccine (plasmid vector). The DCGI has permitted them to undertake a phase III clinical trial with a total of 30,000 participants in India. Zydus Cadila plans to boost its ZyCoV-D production capacity to 150 million doses per year. BBV154 is a replication-deficient adenoviral vectored intranasal vaccine created by Washington University School of Medicine in partnership with Bharat Biotech and Precision Virologic, with Bharat Biotech serving as the Indian manufacturer. They started a phase 1 trial (safety and immunogenicity) with adenovirus vectored intranasal vaccination (NCT04751682). Bharat Biotech retains rights in the rest of the world, while Precision Virologics has optioned rights for Europe, the United States, and Japan.

SARS-CoV-2 RBD Bio E COVID-19 (BECOV2A, BECOV2B, $\mathrm{BECOV} 2 \mathrm{C}$, and BECOV2D) is a protein-based vaccine. Baylor College of Medicine, Biological E Limited, and Dynavax Technologies Corporation developed it, and Biological E Limited is the Indian manufacturer. The vaccine candidate has received funding from India's DBT. CEPI will contribute $\$ 5$ million to assist vaccine production. As a result, Biological E Limited may be able to produce 100 million doses by 2021 . The vaccine will be made available for purchase and delivery through the COVAX facility if it is found to be safe, immunogenic, and effective. Genova Biopharmaceuticals is working with HDT Biotech Corporation to develop HGC019, an mRNA vaccine. HGC019, a native mRNA vaccine candidate, is the first of its kind. The drug's safety and immunogenicity were assessed using a mouse and non-human primate model. Unlike the Pfizer-BioNTech mRNA vaccine, HGCO19 can be kept at $2-8^{\circ} \mathrm{C}(\mathrm{BNT} 162 \mathrm{~b} 2)$.

COVI-VAC is a live-attenuated intranasal vaccine developed by Codagenix in the United States and manufactured by the Serum Institute of India. The first phase of the study (safety and immunogenicity) has begun (NCT04619628). In India, SII has started producing COVI-VAC for a phase 1 clinical trial. Mynvax and the Indian Institute of Science collaborated to create the Mynvax COVID-19 vaccine, a mammalian cell-expressed, glycan-engineered, RBD-based subunit vaccine. A highly thermotolerant lyophilized vaccine can be stored for at least 4 weeks $\left(37^{\circ} \mathrm{C}\right)$ without refrigeration. For up to 16 hours, the vaccine can withstand temperatures of up to $70^{\circ} \mathrm{C}$. A variety of animal models have generated encouraging results (mice, guinea pigs, and hamsters). Mynvax does not have a vaccine production facility. Production and human trials are currently being discussed with additional manufacturers. Griffith University in Australia created the live attenuated SARS-CoV-2 vaccine, and Indian Immunologicals manufactures it in India. Indian Immunologicals has signed a research collaboration agreement with Griffith University to develop the vaccine. They want to use the Vero cell platform technology to massproduce the vaccine. COVAXX in the United States developed UB-612, a multitope peptide-based vaccine, and Aurobindo Pharma in India manufactures it. Aurobindo Pharma has signed an exclusive license deal with COVAXX for the development, production, and marketing of the UB-612 vaccine for India and the United Nations International Children's Emergency Fund agency. COVID-19 vaccine production capacity at Aurobindo Pharma is 400-450 million doses. $^{24}$

\section{Prevention}

The World Health Organization recommends certain protective measures regarding Covid-19 awareness and information dissemination through the media is hands should be washed frequently with an alcohol-based hand rub or soap. Hand hygiene is a milestone to control infectious diseases and prevent cross-transmission of microorganisms and reduce the incidence of healthcare-associated infections, improved hand hygiene practice has been recognized as an important public health measure. ${ }^{25}$ One should Keep at least 2 meters distance away from the individual who is coughing or sneezing. Dirty hands should not be used to rub the head, nose, or mouth. While coughing or sneezing one should, cover the mouth and the nose with a bent elbow and use masks or tissue paper if necessary. When there is fever, cough or breathing issue, medical help should be sought as soon as possible. In congested locations like pilgrimage sites, parks, markets, and tourist attractions, traffic should be kept to a minimum. Closure of nurseries, businesses, local and international travel, schools and universities, and other institutions help in preventing the spread of the infection..26 "A vaccine is necessary to end the world's SARS-CoV-2 pandemic. A successful vaccine is safe, effective, durable, and deployable to large populations."

\section{CONCLUSIONS}

People should be aware of COVID-19's severity and take precautions to protect themselves by such as staying at home, getting vaccinated, minimizing social interactions, avoiding public gatherings, keeping a safe distance and wearing suitable masks in public. To summarize, the governments should enact or strengthen sick leave entitlements to reduce the pressure of work while sick, incentivize the industries to improve working conditions, financially support temporary business closures when necessary and provide housing for those who live in cramped or shared housing. Such interventions have the potential to drastically diminish group transmission. These procedures should be taken depending on the COVID-19 rates and risk factors in the area. We 
conclude that masks alone are insufficient to prevent COVID19 and that they must be used in conjunction with physical distancing, hand cleanliness, and vaccination to put an end to the global SARS-CoV-2 pandemic. A successful vaccination is safe, efficacious, long-lasting, and deployable to large populations and one should follow local health authority's recommendations

Financial or other competing interests: None.

Disclosure forms provided by the authors are available with the full text of this article at jemds.com.

\section{REFERENCES}

[1] World Health Organization. Novel Coronavirus (2019$\mathrm{nCoV}$ ): situation report 3 . World Health Organization 2020.

[2] Vagga AA, Butola LK, Khadhe SG, et al. Association of Natural Antioxidants and Immunity in Covid-19 Pandemic. J Evol Med Dent Sci 2021;10(9):613-9.

[3] Butola LK, Ambad R, Kute PK, et al. The Pandemic of 21st Century-COVID-19. J Evol Med Dent Sci 2020;9(39):2913-9.

[4] Jackson LA, Anderson EJ, Rouphael NG, et al. An mRNA vaccine against SARS-CoV-2-preliminary report. $\mathrm{N}$ Engl J Med 2020;383:1920-31.

[5] Wang F, Kream RM, Stefano GB. An evidence based perspective on mRNA-SARS-CoV-2 vaccine development. Med Sci Monit 2020;26:e924700-1-8.

[6] Pardi N, Hogan MJ, Porter FW, et al. mRNA vaccines - a new era in vaccinology. Nat Rev Drug Discov 2018;17(4):261-79.

[7] Shirley JL, de Jong YP, Terhorst C, et al. Immune responses to viral gene therapy vectors. Mol Ther 2020;28(3):709-22.

[8] Graham SP, McLean RK, Spencer AJ, et al. Evaluation of the immunogenicity of prime-boost vaccination with the replication-deficient viral vectored COVID-19 vaccine candidate ChAdOx1 nCoV-19. NPJ Vaccines 2020;5:69.

[9] Gary EN, Weiner DB. DNA vaccines: prime time is now. Curr Opin Immunol 2020;65:21-7.

[10] Petersen E. Advancing COVID-19 vaccines - avoiding different regulatory standards for different vaccines and need for open and transparent data sharing. Int J Infect Dis 2020;98:501-2.

[11] Tian JH, Patel N, Haupt R, et al. SARS-CoV-2 spike glycoprotein vaccine candidate NVX-CoV2373 elicits immunogenicity in baboons and protection in mice. Nature Communications 2020;12:372.

[12] Wrapp D, Wang N, Corbett KS, et al. Cryo-EM structure of the 2019-nCoV spike in the prefusion conformation. Science 2020;367(6483):1260-3.

[13] Reimer JM, Karlsson KH, Lövgren-Bengtsson K, et al. Matrix- $\mathrm{M}^{\mathrm{TM}}$ adjuvant induces local recruitment, activation and maturation of central immune cells in absence of antigen. PloS One 2012;7(7):e41451.

[14] Li L, Petrovsky N. Molecular mechanisms for enhanced DNA vaccine immunogenicity. Expert Rev Vaccines 2016;15(3):313-29.

[15] Jeyanathan M, Afkhami S, Smaill F, et al. Immunological considerations for COVID-19 vaccine strategies. Nat Rev Immunol 2020;20(10):615-632.

[16] Xia S, Duan K, Zhang Y, et al. Effect of an inactivated vaccine against SARS-CoV-2 on safety and immunogenicity outcomes: interim analysis of 2 randomized clinical trials. JAMA 2020;324(10):951-60.

[17] Xia S, Zhang Y, Wang Y, et al. Safety and immunogenicity of an inactivated SARS-CoV-2 vaccine, BBIBP-CorV: a randomised, double-blind, placebo-controlled, phase $1 / 2$ trial. Lancet Infect Dis 2020;21(1):39-51.

[18] Dutta AK. Vaccine against Covid-19 disease-present status of development. Indian J Pediatr 2020;87(10):810-6.

[19] Dai X, Xiong Y, Li N, Jian C. Vaccine Types Vaccines-The History and Future 2019.

[20] Ng WH, Liu X, Mahalingam S. Development of vaccines for SARS-CoV-2. F1000 Res 2020;9:F1000.

[21] Kaur SP, Gupta V. COVID-19 Vaccine: a comprehensive status report. Virus Res 2020;288:198114.

[22] Kumar VM, Pandi-Perumal SR, Trakht I, et al. Strategy for COVID-19 vaccination in India: the country with the second highest population and number of cases. NPJ Vaccines 2021;6(1):1-7.

[23] Bagcchi S. The world's largest COVID-19 vaccination campaign. Lancet Infect Dis 2021;21(3):323.

[24] Sharun K, Dhama K. India's role in COVID-19 vaccine diplomacy. J Travel Med 2021;28(7):taab064.

[25] Kanyal D, Butola LK. Knowledge, Attitude and practice regarding hand hygiene among medical students and nurses - a questionnaire based survey in tertiary care hospital. International Journal of Research in Pharmaceutical Sciences 2020;11(SPL4):2726-34.

[26] World Health Organisation. Coronavirus. WHO 2020 https:// www. who. Int/ healthtopics/coronavirus. 